\title{
Uso e autoeficácia do preservativo masculino: um estudo comparativo entre adolescentes do sexo feminino e masculino
}

\author{
Male condom use and self-efficacy: a comparative study between female and male adolescents \\ Uso y autoeficácia del condón masculino: un estudio comparativo entre adolescentes femenino y \\ masculino
}

Recebido: 23/11/2021 | Revisado: 02/12/2021 | Aceito: 06/12/2021 | Publicado: 16/12/2021

\author{
Jéssica Aparecida da Silva \\ ORCID: https://orcid.org/0000-0002-4308-5978 \\ Universidade Federal do Triângulo Mineiro, Brasil \\ E-mail: jessicaapssilva@hotmail.com \\ Raquel Pan \\ ORCID: https://orcid.org/0000-0003-1312-3889 \\ Universidade Federal do Triângulo Mineiro, Brasil \\ E-mail: raquel.pan@uftm.edu.br \\ Bethania Ferreira Goulart \\ ORCID: https://orcid.org/0000-0003-2855-6767 \\ Universidade Federal do Triângulo Mineiro, Brasil \\ E-mail: bethaniagoulart@yahoo.com.br \\ Mariana Torreglosa Ruiz \\ ORCID: https://orcid.org/0000-0002-5199-7328 \\ Universidade Federal do Triângulo Mineiro, Brasil \\ E-mail: mariana.ruiz@uftm.edu.br \\ Bibiane Dias Miranda Parreira \\ ORCID: https://orcid.org/0000-0001-7369-5745 \\ Universidade Federal do Triângulo Mineiro, Brasil \\ E-mail: bibiane.parreira@uftm.edu.br
}

\begin{abstract}
Resumo
Objetivo: Caracterizar os adolescentes do sexo feminino e masculino quantos às variáveis comportamentais e de uso de métodos contraceptivos e identificar e associar o escore da autoeficácia no uso de preservativo masculino entre os adolescentes do sexo feminino e masculino de uma escola pública. Metodologia: Este é um estudo descritivo, transversal com abordagem quantitativa, com 95 adolescentes matriculados nos turnos vespertino e noturno de uma escola pública do interior de Minas Gerais, realizado no mês de fevereiro de 2020. Resultados: O preservativo masculino foi o método de escolha mais utilizado, tanto por meninas (27,6\%), quanto por meninos $(90 \%)$. O escore médio dos entrevistados em relação à autoeficácia entre o sexo masculino foi de 58,8 pontos (dp=6,4); em relação às adolescentes do sexo feminino, foram de 58,2 pontos $(\mathrm{dp}=8,3)$. $\mathrm{Na}$ análise bivariada, as variáveis: relação sexual $(p=0,88 ; p=0,13)$, trabalho $(p=0,80 ; p=0,88)$, atividade física $(p=0,89 ; p=0,08)$ e atividade de lazer $(p=0,79 ; p=0,87)$, não estiveram associadas a maior escore da autoeficácia do uso de preservativos entre os adolescentes. Conclusão: Conclui-se que os adolescentes apresentaram boa autoeficácia em relação ao uso do preservativo masculino, e mostrou que não está relacionada a algumas variáveis que poderiam potencializar as evidências.

Palavras-chave: Adolescente; Doenças sexualmente transmissíveis; Gravidez na adolescência; Preservativos; Comportamento do adolescente; Saúde do adolescente.

Abstract

Objective: To characterize female and male adolescents regarding behavioral variables and use of contraceptive methods, and identify and associate the self-efficacy score in male condom use among female and male adolescents at a public school. Methodology: This is a descriptive, cross-sectional study with a quantitative approach, with 95 adolescents enrolled in the afternoon and night shifts of a public school in the interior of Minas Gerais, carried out in February 2020. Results: The male condom was the method of choice most used by both girls (27.6\%) and boys (90\%). The average score of respondents in relation to self-efficacy among males was 58.8 points (sd=6.4); in relation to female adolescents, they were 58.2 points $(\mathrm{sd}=8.3)$. In the bivariate analysis, the variables: sexual intercourse $(\mathrm{p}=0.88$; $\mathrm{p}=0.13)$, work $(\mathrm{p}=0.80 ; \mathrm{p}=0.88)$, physical activity $(\mathrm{p}=0.89 ; \mathrm{p}=0.08)$ and leisure activity $(\mathrm{p}=0.79 ; \mathrm{p}=0.87)$ were not associated with a higher self-efficacy score in condom use among adolescents. Conclusion: It is concluded that adolescents showed good self-efficacy in relation to the use of male condoms, and showed that it is not related to some variables that could enhance the evidence.
\end{abstract}


Keywords: Adolescent; Sexually transmitted diseases; Pregnancy in adolescence; Condoms; Adolescent behavior; Adolescent health.

\begin{abstract}
Resumen
Objetivo: Caracterizar a adolescentes y adolescentes en cuanto a variables de comportamiento y uso de métodos anticonceptivos, e identificar y asociar el puntaje de autoeficacia en el uso de condón masculino entre adolescentes y adolescentes de una escuela pública. Metodología: Se trata de un estudio descriptivo, transversal con enfoque cuantitativo, con 95 adolescentes matriculados en los turnos de tarde y noche de un colegio público del interior de Minas Gerais, realizado en febrero de 2020. Resultados: El condón masculino fue el método de elección más utilizado tanto por niñas $(27,6 \%)$ como por niños $(90 \%)$. La puntuación media de los encuestados en relación con la autoeficacia entre los hombres fue de 58,8 puntos $(\mathrm{dt}=6,4)$; en relación a las mujeres adolescentes, fueron 58,2 puntos $(\mathrm{dt}=8,3)$. En el análisis bivariado, las variables: relaciones sexuales $(\mathrm{p}=0,88 ; \mathrm{p}=0,13)$, trabajo $(\mathrm{p}=0,80 ; \mathrm{p}=$ $0,88)$, actividad física $(p=0,89 ; p=0,08)$ y actividad de ocio $(p=0,79 ; p=0,87)$ no se asociaron con una puntuación más alta de autoeficacia en el uso del condón entre los adolescentes. Conclusión: Se concluye que los adolescentes mostraron buena autoeficacia en relación al uso del condón masculino, y demostraron que no se relaciona con algunas variables que podrían potenciar la evidencia.
\end{abstract}

Palabras clave: Adolescente; Enfermedades de transmisión sexual; Embarazo en adolescencia; Condones; Conducta del adolescente; Salud del Adolescente.

\title{
1. Introdução
}

A adolescência segundo o Ministério da Saúde (2010), compreende o período entre 10 e 19 anos, 11 meses e 29 dias de idade, onde ocorre o crescimento, o desenvolvimento e a transição para a vida adulta, acompanhado de transformações físicas, psíquicas e emocionais (Rodrigues et al., 2018).

Dentre todas as mudanças que os adolescentes passam, surge o interesse a respeito das relações sexuais, consideradas um componente intrínseco da pessoa e fundamental na saúde, não sendo um fator apenas biológico, mas também psicológico e social, muitas vezes influenciado pelas crenças e valores pessoais, família e sociedade (Brasil, 2010).

Diante disso, torna-se fundamental ter orientações prévias para que o adolescente se sinta seguro e autônomo diminuindo os riscos de gerar consequências, como, uma gravidez indesejada ou até mesmo contrair infecção sexualmente transmissível (IST). Esses fatores são considerados como um problema de saúde pública, que se repete por gerações entre as classes mais pobres (Rodrigues et al., 2018).

O início das relações sexuais de forma precoce e com o uso inconsistente do preservativo são comportamentos que deixam o adolescente numa situação de maior vulnerabilidade e gera consequências pela falta de proteção durante o ato sexual (Krabbe et al., 2017; Silva et al., 2015).

Segundo o Ministério da Saúde, a camisinha é o método mais eficaz para se prevenir contra muitas IST’s (Brasil, 2017). Uma pesquisa realizada em Pelotas mostrou que $18 \%$ dos adolescentes do sexo feminino e masculino não utilizam preservativos nas relações sexuais (Miranda et al., 2018). Em 2015, 27,5\% dos alunos do último ano do fundamental disseram já ter tido relação sexual alguma vez e 33,8\% dos jovens não tinham usado preservativo (Brasil, 2015). As crenças e atitudes em relação ao preservativo, de que o mesmo causa desconforto e interfere no prazer, ainda perdura entre os jovens e contribuem com a vulnerabilidade desta população (Valim, Dias, Simon, Almeida \& Rodrigues, 2015). A questão de gênero também é um fator que está diretamente relacionada ao não uso do preservativo, pois muitas mulheres não conseguem impor ao seu parceiro a utilização do mesmo, além de correr o risco de ser julgada caso tenha um preservativo na bolsa (Krabbe et al., 2017).

Um estudo realizado em Parelhas-RN mostrou que a maioria dos adolescentes possui conhecimento geral inadequado, relacionado a algumas práticas, sendo principalmente a não adesão ao uso do preservativo (Cordeiro et al., 2017). Por outro lado, um estudo realizado em Cruz Alta-RS mostrou que os adolescentes tinham conhecimento sobre a importância do uso dos preservativos, porém não aplicavam na prática, resultando em comportamentos de risco constantes tanto para contraírem IST's, quanto para gravidez indesejada (Krabbe et al., 2017). 
É na adolescência que necessitam de maior nível de atenção e orientação frente aos problemas e vulnerabilidades (Cordeiro et al., 2017), portanto é importante a partir de implementação de políticas públicas um cuidado integral envolvendo aspectos físicos, mentais e sociais, incluindo a educação sexual que pode ser discutida por profissionais de saúde, educadores e família, envolvendo a todos a responsabilidade de um desenvolvimento saudável e adequado para o adolescente (Silva et al., 2015), visto que são considerados uma população vulnerável às ISTs e gravidez indesejada (Rodrigues et al., 2018). Deve-se considerar então, as atitudes tomadas em relação a sexualidade que podem gerar impactos na saúde desses adolescentes.

Diante disso, indaga-se se o conhecimento sobre os métodos contraceptivos, especificamente o preservativo está sendo repassado, como está sendo repassado e, se este método está sendo utilizado na prática. Visto a importância da temática para a saúde sexual e reprodutiva desta faixa etária, atos e decisões no período da adolescência são fundamentais e podem impactar de várias formas na qualidade de vida, familiar e profissional desses adolescentes. A realidade exposta anteriormente evidencia a importância da realização de novas pesquisas na área, comparando com outros estudos e analisando a diversidade de dados e informações que poderão contribuir para as ações em diversos âmbitos e setores, principalmente para mudanças nas políticas públicas do município, nas ações dentro da escola, com a família e na atuação dos profissionais de saúde. Esses, com destaque para o enfermeiro, podem e devem desenvolver atividades assistenciais e educativas voltadas para os adolescentes dentro das escolas.

Este trabalho teve como objetivo: Caracterizar os adolescentes do sexo feminino e masculino quantos às variáveis comportamentais e de uso de métodos contraceptivos e identificar e associar o escore da autoeficácia no uso de preservativo masculino entre os adolescentes do sexo feminino e masculino de uma escola pública.

\section{Metodologia}

Este é um estudo quantitativo com abordagem descritiva e transversal, do qual trabalha com indicadores numéricos e segue critérios estáticos, descrevendo os fenômenos e avaliando a frequência e distribuição de variáveis de estudo em um determinado grupo demográfico em um único momento (Pereira et al., 2018; Zangirolami-Raimundo et al., 2018).

Foi realizado em uma cidade no interior de Minas Gerais. O município possui um total de 513 escolas estaduais e municipais, sendo 74 rurais e 439 urbanas; destas apenas 23 contam com ensino médio (SRE, 2019). Para o presente estudo foi selecionada a escola com maior número de estudantes. Os sujeitos deste estudo foram alunos do $1^{\circ}$ ao $3^{\circ}$ ano do ensino médio dos turnos vespertino e noturno, de ambos os sexos, que estavam presentes no momento da coleta de dados.

A coleta de dados ocorreu no mês de fevereiro de 2020, em horários estabelecidos pela escola em dois turnos, sendo tarde e noite. Em um primeiro momento, foi explicado os objetivos do trabalho e entregue para 246 alunos os termos de consentimento livre e esclarecido (TCLE), para maiores de 18 anos, o termo de assentimento, e o termo de consentimento do responsável legal, para os pais dos menores de idade. Em um segundo momento, foi aplicado um instrumento auto preenchível para aqueles que devolveram o TCLE assinado, sendo 62 alunos do turno vespertino e 33 do turno noturno, gerando um total de 95 alunos e todos estes devolveram os questionários respondidos.

Os instrumentos continham um questionário elaborado, abordando questões sociodemográficas, econômicas, ginecológicas e obstétricas com 20 questões e a Escala de Autoeficácia no Uso de Preservativos traduzida e adaptada para a língua portuguesa no contexto brasileiro, composta por 14 itens que avaliam comunicação, uso consistente e correto do preservativo. São mensurados em escala tipo Likert, que varia de 1 a 5, na qual 1 representa muito inseguro, 2 inseguro, 3 pouco seguro, 4 seguro e 5 muito seguro (Sousa et al., 2018).

A análise dos dados ocorreu a partir da digitação dos dados em uma planilha eletrônica no programa EXCEL e posteriormente transportadas para o "SPSS Statistical Analysis Software" versão 1.7., para a análise uni variada dos dados, foi 
utilizado a distribuição de frequências absolutas (n) e relativas (\%) e valores de média e desvios-padrão e na análise bivariada foi usado o Teste t-Student.

Esta pesquisa faz parte de um projeto amplo titulado "Sexualidade e autoeficácia no uso de preservativos dos adolescentes de uma escola pública", que foi aprovado pelo Comitê de Ética em Pesquisa (CEP) da Universidade Federal do Triângulo Mineiro via Plataforma Brasil (CAAE. 05196818.7.0000.5154) a fim de cumprir as exigências da Resolução 466/12 do Conselho Nacional de Saúde que regulamenta as normas para a realização de pesquisas que envolvem seres humanos. Como também foi solicitada a autorização da escola em questão para participação no estudo.

\section{Resultados}

Em relação às características sociodemográficas, participaram do estudo 51 adolescentes do sexo feminino e 44 do sexo masculino, tendo o predomínio de participantes do sexo feminino (53,7\%), com idade média de 16 anos entre as meninas e 17 anos entre os meninos, e status marital solteiro (70,6\% e 77,3\% respectivamente). Quanto ao grau de escolaridade dos participantes, $23,5 \%$ e $31,8 \%$ estavam matriculados no primeiro ano do ensino médio, 29,4\% e 38,6\% no segundo ano e $47,1 \%$ e $29,5 \%$ no $3^{\circ}$ ano, do sexo feminino e masculino respectivamente.

Das características econômicas dos participantes, destacou-se que 37,3\% do sexo feminino e 45,5\% do sexo masculino continham ocupação remunerada. Quanto à distribuição da renda familiar, a maioria não tinha conhecimento sobre os valores $(84,3 \% ; 81,8 \%)$ e em relação ao número de pessoas residentes no domicílio, 74,5\% e 72,7\% moravam com até 4 pessoas.

Sobre o comportamento diário dos participantes, $58,8 \%$ do sexo feminino e $29,5 \%$ do sexo masculino não praticavam atividade física, porém houve predomínio na realização de atividades de lazer (84,3\% e 86,4\%). Alguns hábitos chamaram a atenção, como fumar (3,9\% e 18,2\%), consumir bebidas alcóolicas $(62,8 \%$ e 38,6\%) e uso de drogas ilícitas (4\% e 9,1\%).

Em relação ao comportamento sexual, 60,8\% das meninas e 61,4\% dos meninos já haviam tido relação sexual. Destes, respectivamente $6,5 \%$ e $26 \%$ não utilizavam qualquer tipo de método contraceptivo. Entre os que utilizavam, os mais recorridos encontram-se na Tabela 1.

Tabela 1 - Métodos contraceptivos mais utilizados entre os adolescentes do sexo feminino e masculino com vida sexual ativa. 2020 .

\begin{tabular}{lcc}
\hline Variáveis & $\begin{array}{c}\text { Sexo Feminino } \\
\text { \% }\end{array}$ & $\begin{array}{c}\text { Sexo Masculino } \\
\text { \% }\end{array}$ \\
\hline \hline Preservativo masculino & 27,6 & 90 \\
Preservativo masculino e hormonal oral & 13,8 & 0 \\
Hormonal oral & 13,8 & 0 \\
Preservativo masculino, hormonal oral, coito interrompido e pílula de & 10,3 & 0 \\
emergência & 7 & 0 \\
Pílula de emergência & 10,3 & 0 \\
Hormonal injetável & 0 & 5 \\
Preservativo masculino e coito interrompido & 0 & 5 \\
Preservativo masculino e feminino & 17,2 & 0 \\
Outros & & 5 \\
\hline
\end{tabular}

Fonte: Autores (2020).

O preservativo masculino foi o método de escolha mais utilizado, tanto por meninas, quanto por meninos e em seguida, a combinação do preservativo masculino com hormonal oral $(13,8 \%)$ entre as meninas. Com relação à orientação 
sobre a utilização dos métodos contraceptivos destacou, entre as meninas o profissional médico $(44,8 \%)$ e entre os meninos o pai (35\%). Predominantemente os participantes referiram que conseguem os métodos na farmácia $(82,8 ; 75 \%)$ e apenas $10,3 \%$ das meninas e 15\% dos meninos adquirem os métodos através das unidades de saúde.

Neste estudo, $11,1 \%$ dos participantes do sexo masculino já haviam se tornado pais na adolescência, tal ocorrido entre as idades de 15 e 19 anos e dois terços destas gestações não foram planejadas. Já entre as participantes do sexo feminino não houve gestações.

No que tange a escala de autoeficácia no uso de preservativos, aplicada aos adolescentes, a referida escala continha perguntas relacionadas à segurança e insegurança as ações relacionadas ao uso do preservativo masculino. As respostas foram analisadas por meio de porcentagem (Tabela 2).

Tabela 2 - Porcentagem de respostas sobre a escala de autoeficácia no uso de preservativos entre os adolescentes do sexo feminino e masculino. 2020.

\section{ESCALA DE AUTOEFICÁCIA NO USO DE PRESERVATIVOS - VERSÃO BRASILEIRA}

\begin{tabular}{|c|c|c|c|c|c|c|c|c|c|c|}
\hline & \multicolumn{5}{|c|}{ Sexo Feminino } & \multicolumn{5}{|c|}{ Sexo Masculino } \\
\hline & $\begin{array}{l}\text { Muito } \\
\text { inseguro }\end{array}$ & Inseguro & $\begin{array}{l}\text { Pouco } \\
\text { seguro }\end{array}$ & Seguro & $\begin{array}{l}\text { Muito } \\
\text { seguro }\end{array}$ & $\begin{array}{l}\text { Muito } \\
\text { inseguro }\end{array}$ & Inseguro & $\begin{array}{l}\text { Pouco } \\
\text { seguro }\end{array}$ & Seguro & $\begin{array}{l}\text { Muito } \\
\text { seguro }\end{array}$ \\
\hline & \multicolumn{5}{|c|}{$\%$} & \multicolumn{5}{|c|}{$\%$} \\
\hline $\begin{array}{l}\text { Eu posso ter preservativo comigo caso eu } \\
\text { necessitar usar. }\end{array}$ & 11,8 & 11,8 & 2 & 33,3 & 41,2 & 6,8 & 4,5 & 18,2 & 36,4 & 34,1 \\
\hline $\begin{array}{l}\text { Eu posso usar preservativo toda vez que eu e meu } \\
\text { (minha) parceiro(a) tivemos relação sexual. }\end{array}$ & 5,9 & 0 & 7,8 & 21,6 & 64,7 & 0 & 0 & 2,3 & 47,7 & 50 \\
\hline $\begin{array}{l}\text { Eu posso usar um novo preservativo toda vez que } \\
\text { eu e meu (minha) parceiro(a) tivemos relação } \\
\text { sexual. }\end{array}$ & 3,9 & 3,9 & 2 & 33,3 & 56,9 & 0 & 0 & 2,3 & 27,3 & 70,5 \\
\hline $\begin{array}{l}\text { Eu posso parar o envolvimento sexual antes de } \\
\text { qualquer penetração para colocar o preservativo } \\
\text { em mim ou em meu(minha) parceiro(a) sexual. }\end{array}$ & 3,9 & 9,8 & 7,8 & 35,3 & 43,1 & 2,3 & 6,8 & 6,8 & 34,1 & 50 \\
\hline $\begin{array}{l}\text { Eu e o meu(minha) parceiro(a) podemos colocar } \\
\text { o preservativo desenrolando-o até a base do } \\
\text { pênis. }\end{array}$ & 7,8 & 9,8 & 21,6 & 17,6 & 43,1 & 0 & 9,1 & 4,5 & 34,1 & 52,3 \\
\hline $\begin{array}{l}\text { Eu e o meu(minha) parceiro(a) podemos colocar } \\
\text { o preservativo no pênis depois de ereto para } \\
\text { assim não escorregar. }\end{array}$ & 3,9 & 7,8 & 13,7 & 41,2 & 33,3 & 0 & 4,5 & 11,4 & 29,5 & 54,5 \\
\hline $\begin{array}{l}\text { Eu e o meu(minha) parceiro(a) podemos segurar } \\
\text { o preservativo contra a base do pênis durante a } \\
\text { sua retirada após a relação sexual. }\end{array}$ & 7,8 & 13,7 & 17,6 & 23,5 & 37,3 & 2,3 & 6,8 & 15,9 & 38,6 & 36,4 \\
\hline $\begin{array}{l}\text { Eu e o meu(minha) parceiro(a) podemos colocar } \\
\text { o preservativo no lixo, após a relação sexual. }\end{array}$ & 3,9 & 7,8 & 3,9 & 35,3 & 49 & 4,5 & 6,8 & 9,1 & 31,8 & 47,7 \\
\hline $\begin{array}{l}\text { Eu e o meu(minha) parceiro(a) podemos usar o } \\
\text { preservativo após ingerir bebida alcoólica. }\end{array}$ & 9,8 & 11,8 & 11,8 & 19,6 & 47,1 & 4,5 & 0 & 29,5 & 25 & 40,9 \\
\hline $\begin{array}{l}\text { Eu posso conversar sobre o uso do preservativo } \\
\text { com qualquer parceiro(a) sexual. }\end{array}$ & 3,9 & 3,9 & 3,9 & 11,8 & 76,5 & 0 & 0 & 6,8 & 31,8 & 61,4 \\
\hline $\begin{array}{l}\text { Eu posso conversar sobre o uso do preservativo, } \\
\text { mesmo se eu tiver inseguro(a) da opinião do } \\
\text { meu(minha) parceiro(a) sobre preservativo. }\end{array}$ & 5,9 & 2 & 2 & 29,4 & 60,8 & 0 & 4,5 & 9,1 & 34,1 & 52,3 \\
\hline $\begin{array}{l}\text { Eu posso conversar sobre o uso do preservativo } \\
\text { com um possível parceiro(a) sexual antes de } \\
\text { começarmos a nos abraçar e beijar. }\end{array}$ & 5,9 & 3,9 & 11,8 & 31,4 & 47,1 & 6,8 & 6,8 & 27,3 & 27,3 & 31,8 \\
\hline $\begin{array}{l}\text { Eu posso convencer um (a) parceiro(a) a usar } \\
\text { preservativo no momento da relação sexual. }\end{array}$ & 2 & 2 & 3,9 & 19,6 & 72,5 & 2,3 & 4,5 & 11,4 & 25 & 56,8 \\
\hline $\begin{array}{l}\text { Eu posso recusar o sexo se meu(minha) } \\
\text { parceiro(a) se recusar a usar preservativo. }\end{array}$ & 2 & 2 & 2 & 9,8 & 84,3 & 13,6 & 2,3 & 11,4 & 20,5 & 52,3 \\
\hline
\end{tabular}

Fonte: Autores (2020). 
O escore médio dos entrevistados do sexo masculino foi de 58,8 pontos $(\mathrm{dp}=6,4)$ e mediana de 59,0 pontos, com variação entre 41 e 70, já entre adolescentes do sexo feminino o escore médio foi de 58,2 pontos (dp=8,3) e mediana de 61,0 pontos, com variação entre 34 e 70. Na análise bivariada, foram analisadas a correlação do maior escore da autoeficácia do uso de preservativos entre os adolescentes e algumas características comportamentais e econômica (Tabela 3).

Tabela 3 - Comparação das variáveis econômicas e comportamentais sobre a autoeficácia do uso de preservativos entre os adolescentes do sexo masculino e feminino. 2020.

\begin{tabular}{|c|c|c|c|c|c|c|}
\hline \multirow{2}{*}{ VARIÁVEIS } & \multicolumn{2}{|c|}{ SEXO MASCULINO } & \multirow[b]{2}{*}{$p^{*}$} & \multicolumn{3}{|c|}{ SEXO FEMININO } \\
\hline & Média & Desvio- padrão & & Média & Desvio- padrão & $p^{*}$ \\
\hline Relação Sexual & & & 0,88 & & & 0,13 \\
\hline $\operatorname{Sim}$ & 58,7 & 5,9 & & 59,7 & 7,2 & \\
\hline Não & 59,0 & 7,3 & & 56,1 & 9,5 & \\
\hline Trabalho & & & 0,80 & & & 0,88 \\
\hline $\operatorname{Sim}$ & 58,5 & 6,4 & & 58,5 & 8,7 & \\
\hline Não & 59,0 & 6,6 & & 58,1 & 8,2 & \\
\hline Atividade Física & & & 0,89 & & & 0,08 \\
\hline Sim & 58,9 & 7,0 & & 60,7 & 6,9 & \\
\hline Não & 58,6 & 4,8 & & 56,6 & 8,9 & \\
\hline Atividade de Lazer & & & 0,79 & & & 0,87 \\
\hline $\operatorname{Sim}$ & 58,9 & 6,6 & & 58,3 & 8,0 & \\
\hline Não & 58,1 & 5,4 & & 57,8 & 10,3 & \\
\hline
\end{tabular}

*valor de p para o Teste t Student. Fonte: Autores (2020).

As variáveis: relação sexual $(p=0,88 ; p=0,13)$, trabalho $(p=0,80 ; p=0,88)$, atividade física $(p=0,89 ; p=0,08)$, e atividade de lazer $(p=0,79 ; p=0,87)$, não estiveram associadas a maior escore da autoeficácia do uso de preservativos entre os adolescentes, feminino e masculino, respectivamente.

\section{Discussão}

No grupo estudado, a maioria dos participantes era do sexo feminino (53,7\%), com idade média de 16 e 17 anos, entre os do sexo feminino e masculino respectivamente, predominando a situação conjugal solteiro para ambos os sexos (70,6\%; 77,3\%). Tais dados corroboram aos achados de outros trabalhos nacionais e internacionais que verificaram perfis similares (Silva \& Lopes, 2018; Mola et al., 2016; Moyo \& Rusinga, 2017; Eggers et al., 2016; Xu et al., 2017; Nunes et al., 2017; Krabbe et al., 2017).

Das características econômicas, destacou que 37,3\% e 45,5 dos adolescentes já possuíam ocupação remunerada, demonstrando que muitos jovens se inserem no mercado de trabalho para contribuir com a renda familiar e adquirir independência financeira (Amazarray et al., 2009).

Sobre o comportamento dos participantes no dia-a-dia, $58,8 \%$ do grupo feminino e $29,5 \%$ do masculino não praticavam atividade física, sendo relevante se atentar a este índice, tendo em vista a importância do desenvolvimento de hábitos de vida saudáveis entre os jovens, visando a sua manutenção na idade adulta (Dumith et al., 2012), por outro lado houve predomínio na realização de atividades de lazer $(84,3 \%$ e $86,4 \%$ ), o que pode ser um fator importante para a troca de informações ao encontrar amigos e familiares durante estas atividades. Outros hábitos também chamaram a atenção, como fumar (3,9\% e 18,2\%), consumir bebidas alcóolicas (62,8\% e 38,6\%) e uso de drogas ilícitas (4\% e 9,1\%), tais dados também aparecem em alguns estudos (Almeida, 2017; Oliveira et al., 2019) e podem ser explicados devido ao interesse dos adolescentes em ter novas experiências. É importante considerar que apesar das mudanças e experiências nessa etapa de desenvolvimento, algumas ações podem afetar e trazer consequências para a vida adulta (García \& Costa Junior, 2008). 
Em relação ao comportamento sexual, $60,8 \%$ do sexo feminino e $61,4 \%$ do sexo masculino já haviam tido relação sexual, tal acontecimento entre os adolescentes também aparece em outros estudos (Moyo \& Rusinga, 2017; Silva \& Lopes, 2018; Genz et al., 2017; Nunes et al., 2017; Young et al., 2018), demonstrando que durante a adolescência surgem as primeiras curiosidades a respeito do sexo e é nesse momento que muitos jovens tem sua primeira relação sexual. Portanto, deve ser um assunto encarado como um componente intrínseco e abordado de forma cotidiana para que não haja comportamentos inadequados, inseguros e práticas sexuais de risco (Oliveira et al., 2017; Krabbe et al., 2017). Um fato interessante foi a proximidade do percentual de prática sexual entre meninas e meninos no presente estudo. Esse fato demonstra uma mudança cultural em que existia uma diferença entre meninas e meninos no início da prática sexual na adolescência, visto que o homem era precocemente estimulado a ter relações sexuais, enquanto a mulher era desencorajada (Tavares et al., 2009).

Destaca-se que, destes 6,5\% das meninas e $26 \%$ dos meninos não utilizavam qualquer tipo de método contraceptivo, sendo um fator de risco para a gravidez não desejada. Compreende-se que a idade e a inexperiência associada podem implicar nas atitudes em relação aos preservativos, que ainda estão em desenvolvimento junto com a experiência do sexo (Eggers et al., 2016).

Por outro lado, os adolescentes que utilizavam algum método contraceptivo, o mais recorrido foi o preservativo masculino, utilizado tanto por meninas $(27,6 \%)$, quanto por meninos $(90 \%)$ e em seguida, a combinação do preservativo masculino com hormonal oral $(13,8 \%)$ entre as meninas. Um estudo também realizado em uma cidade do interior de Minas Gerais, com 1193 adolescentes, identificou resultados parecidos, o uso do preservativo masculino foi predominante (66,6\%), seguido da combinação do preservativo masculino e pílula anticoncepcional (8\%) (Silva \& Lopes, 2018).

Com relação à orientação sobre a utilização dos métodos contraceptivos, destacou-se entre as meninas, o médico $(44,8 \%)$ e entre os meninos o pai (35\%), podendo ser fatores positivos, visto que são fontes seguras, porém os pais também podem trazer conceitos errôneos, com poucas informações e abertura para o diálogo. Um estudo realizado em Porto AlegreRS, concluiu que a comunicação sobre sexualidade recebida pela família foi predominantemente avaliada como precária pelos adolescentes, especialmente devido ao constrangimento daqueles que se propõem a dialogar sobre o tema e em sua maioria, as conversas costumavam ser limitadas a informações pontuais, por vezes com tom de ameaça (Furlanetto et al., 2019).

Quando estes adolescentes não conseguem informações por profissionais ou familiares, eles acabam recorrendo aos amigos e a internet, o que também pode ser um problema, uma vez que, o círculo social do adolescente, também é composto por outros adolescentes que ainda estão em processo de formação do conhecimento, como também a internet pode ter sites não confiáveis, fazendo com que as informações sejam transmitidas de forma errônea (Krabbe et al., 2017).

Os profissionais de saúde têm papel fundamental em relação à educação em saúde voltada para as questões de saúde sexual e reprodutiva. Eles precisam expandir suas competências dentro dessa temática, tornando-se ponto de referência para esses adolescentes que necessitam de orientações e assistência em saúde. Os grupos educativos nas escolas, na atenção primária e as ações específicas em saúde, como as consultas, fazem com que os profissionais de saúde tenham um papel imprescindível junto aos adolescentes, oferecendo suporte e informações adequadas.

Predominantemente os adolescentes conseguem os métodos através da farmácia (82,8\%; 75\%), apenas 10,3\% e 15\% adquirem os métodos através das unidades de saúde. Tal realidade também acontece em outros países, como por exemplo, no estudo realizado em Zimbabwe na África com 185 adolescentes. O estudo identificou que as unidades de saúde tiveram apenas 0,3\% dos adolescentes acessando anticoncepcionais, além disso, a maioria dos entrevistados (79\%), afirmou que não se sentiria à vontade para obter anticoncepcionais em um centro de saúde, sendo as mulheres 88\%, e os homens 59\% (Moyo \& Rusinga, 2017). Estes resultados podem ser explicados pelo pré-julgamento enraizado da sociedade em relação ao sexo e a dificuldade de um profissional de referência e a inserção desses adolescentes dentro dos serviços de saúde. 
O presente estudo verificou que 11,1\% participantes do sexo masculino já haviam engravidado alguém, tal ocorrido entre a idade de 15 e 19 anos e dois terços destas gestações não foram planejadas, o que pode ocasionar consequências biológicas, socioeconômicas e culturais (Dias \& Teixeira, 2010).

Com relação a autoeficácia no uso de preservativos, o escore médio dos entrevistados do sexo feminino foi de 58,2 pontos, já entre adolescentes do sexo masculino, 58,8 pontos. O estudo de Sousa et al. (2018), realizado em Fortaleza-CE com 40 participantes, encontrou escores superiores com média da escala de 66,7 entre o sexo feminino e 69,4 do sexo masculino. Mas com a semelhança, com o presente estudo de escore maior entre os meninos. Similar ao estudo realizado em Bahamas, com 1012 participantes. A média da autoeficácia no uso de preservativo foi também maior entre os do sexo masculino do que os do sexo feminino (Xu et al., 2017).

Observou-se na presente pesquisa que a autoeficácia em relação ao uso do preservativo foi um pouco maior entre os meninos. Não apresentando uma diferença tão significativa. Portanto, acredita-se que apesar do uso do preservativo ser de domínio masculino, o escore sobre a autoeficácia foi próxima aos dois sexos. Isso reflete o papel da mulher em relação à sua preocupação em ter práticas sexuais seguras e saudáveis. Embora, medir o uso de preservativos entre adolescentes não é fácil, por mais que se utiliza questionários autoaplicáveis, os resultados podem ser tendenciosos devido a respostas socialmente desejáveis (Eggers et al., 2016).

O maior escore da autoeficácia do uso de preservativos entre os adolescentes não estiveram associados à relação sexual, trabalho, atividade física e atividade de lazer. Essas variáveis poderiam estar relacionadas ao fato desses adolescentes estarem em contato e conversarem sobre o tema com outras pessoas e/ou adolescentes no convívio do trabalho e de atividades física e de lazer. Mas, no entanto, não foi identificado essa associação neste estudo.

A variável ter tido relação sexual não apresentou associação com o maior escore da autoeficácia do uso do preservativo. Portanto, podemos inferir que conhecimento e prática são duas ações que podem não estar associadas, apesar de ser bidirecional - uma pode interligar a outra.

Considera-se indispensável que tanto os adolescentes quanto os envolvidos no seu acompanhamento, conheçam e pratiquem as legislações referentes aos direitos sexuais e reprodutivos, dos quais visam a garantia da vivencia da sexualidade de forma mais orientada e livre de conceitos preestabelecidos pelos padrões morais, religiosos, sociais e culturais.

Por fim, acredita-se que o enfermeiro, como educador, proporciona a criação de um vínculo por meio dessas ações, tanto dentro das instituições de ensino, quanto dentro da unidade de saúde e pode contribuir holisticamente para o empoderamento dos adolescentes e na implementação de medidas preventivas e, consequentemente, na efetivação de ações que possibilitem a redução da vulnerabilidade desses indivíduos as IST e gravidez não planejada ou indesejada.

\section{Conclusão}

Conclui-se que os adolescentes tanto do sexo feminino quanto do sexo masculino apresentaram boa autoeficácia em relação ao uso do preservativo masculino, mas que ainda pode ser melhorado e mostrou que não está relacionada a algumas variáveis que poderiam potencializar as evidências. Além disso, foi evidenciado pelo estudo um alto percentual do uso do preservativo masculino entre os meninos, porém ainda baixo entre as meninas.

Podemos considerar que a idade e a inexperiência associada dos adolescentes podem implicar em conhecimentos e atitudes em direção ao uso do preservativo, pois ainda estão em desenvolvimento junto com a experiência do sexo.

Diante a esta realidade, torna-se fundamental a realização de outros estudos com o objetivo de investigar o uso do preservativo masculino entre os adolescentes e assim contribuir para melhoria de políticas públicas voltadas para esta população a fim de proteger e preservar a saúde e o bem-estar de todos, visto que é um assunto de importante discussão no meio social, já que pode afetar a população e prejudicar as gerações futuras. 
Esta pesquisa pode apresentar fragilidades, uma vez que estes dados foram coletados em apenas uma escola e não se pretende generalizar os resultados. Outra limitação que o estudo apresentou foi referente ao número de participantes, visto que foram entregues os termos de consentimento livre e esclarecido para 246 alunos e apenas 95 alunos devolveram os termos e responderam ao questionário. Além disso, a coleta de dados teve que ser interrompida devido à paralisação das atividades escolares decorrente da pandemia Covid-19.

\section{Referências}

Almeida, N. D. (2017). Uso de álcool, tabaco e drogas por jovens e adultos da cidade de Recife. Psicologia argumento, 29(66). https://periodicos.pucpr.br/index.php/psicologiaargumento/article/view/20285

Amazarray, M. R., Thomé, L. D., Souza, A. P. L. D., Poletto, M., \& Koller, S. H. (2009). Aprendiz versus trabalhador: adolescentes em processo de aprendizagem. Psicologia: Teoria e Pesquisa, 25, 329-338. https://doi.org/10.1590/S0102-37722009000300006

Brasil (2017). Ministério da Saúde. Departamento de Vigilância, Prevenção e Controle das IST, do HIV/Aids e das Hepatites Virais. http://www.aids.gov.br/pagina/por-que-usar

Brasil (2010). Ministério da Saúde. Diretrizes nacionais para a atenção integral à saúde de adolescentes e jovens na promoção, proteção e recuperação da saúde. http://bvsms.saude.gov.br/bvs/publicacoes/diret1JRuYcDcmXWky8CyvW79pq1qxePi72FucuiGE9vhUuKJsFMLz.pdf

Brasil (2015). Ministério da Saúde. Ministério do planejamento, desenvolvimento e gestão. Pesquisa nacional de saúde escolar. https://biblioteca.ibge.gov.br/visualizacao/livros/liv97870.pdf

Cohen J. (1988) Statistical Power Analysis for the Behavioral Sciences (2a ed.) Academic Press. http://www.utstat.toronto.edu/ brunner/oldclass/378f16/readings/CohenPower.pdf

Cordeiro, J. K. R., Santos, M. M. D., Sales, L. K. O., Morais, I. F. D., \& Dutra, G. R. S. D. F. (2017). Adolescentes escolares acerca das DST/AIDS: quando o conhecimento não acompanha as práticas seguras. Rev. Enferm. UFPE online, 2888-2896. https://doi.org/10.5205/reuol.11007-98133-3-SM.1107sup201710

Dias, A. C. G., \& Teixeira, M. A. P. (2010). Gravidez na adolescência: um olhar sobre um fenômeno complexo. Paidéia, 20, 123-131. https://doi.org/10.1590/S0103-863X2954321706485

Dumith, S. C., Gigante, D. P., Domingues, M. R., Hallal, P. C., Menezes, A. M., \& Kohl, H. W. (2012). Predictors of physical activity change during adolescence: a 3. 5-year follow-up. Public Health Nutrition, 15(12), 2237-2245. https://doi.org/10.1017/S1368980012000948

Eggers, S. M., Aarø, L. E., Bos, A. E., Mathews, C., Kaaya, S. F., Onya, H., \& de Vries, H. (2016). Socio cognitive predictors of condom use andintentions among adolescents in three sub-saharan sites. Archivesof sexual behavior, 45(2), 353-365. https://doi.org/10.1007/s10508-015-0525-1

Furlanetto, M. F., Marin, A. H., \& Gonçalves, T. R. (2019). Acesso e qualidade da informação recebida sobre sexo e sexualidade na perspectiva adolescente. Estudos e Pesquisas em Psicologia, 19(3), 644-664. https://doi.org/10.12957/epp.2019.46907

García, K. S. L., \& Costa Junior, M. L. D. (2008). Conduta anti-social e consumo de álcool em adolescentes escolares. Revista Latino-Americana de Enfermagem, 16, 299-305. https://doi.org/10.1590/S0104-11692008000200020

Genz, N., Meincke, S. M. K., Carret, M. L. V., Corrêa, A. C. L., \& Alves, C. N. (2017). Doenças sexualmente transmissíveis: conhecimento e comportamento sexual de adolescentes. Texto \& Contexto-Enfermagem, 26. https://doi.org/10.1590/0104-07072017005100015

Jezo, R. F. V., Ribeiro, I. K. S., Araújo, A., \& Rodrigues, B. A. (2017). Gravidez na adolescência: perfil das gestantes e mães adolescentes em uma unidade básica de saúde. Revista de enfermagem do centro-oeste mineiro, 7. https://doi.org/10.19175/recom.v7i0.1387

Krabbe, E. C., Rodrigues, K. S., Schneider, F. R. L., Bonaldi, J., Baptista, J., Marasca, L., \& Carvalho, T. G. M. L. (2017). Conhecimento, atitudes e práticas com relação ao uso do preservativo no iee professor annes dias. REVISTA INTERDISCIPLINAR DE ENSINO, PESQUISA E EXTENSÃO-RevInt, 5(1). http://revistaeletronica.unicruz.edu.br/index.php/eletronica/article/download/102-111/pdf_133

Miranda, P. S. F., Aquino, J. M. G., Monteiro, R. M. P. D. C., Dixe, M. D. A. C. R., Luz, A. M. B. D., \& Moleiro, P. (2018). Sexual behaviors: study in theyouth. Einstein (São Paulo), 16. https://doi.org/10.1590/S1679-45082018AO4265

Mola, R., Pitangui, A. C. R., Barbosa, S. A. M., Almeida, L. S., Sousa, M. R. M. D., Pio, W. P. D. L., \& Araújo, R. C. D. (2016). Uso de preservativo e consumo de bebida alcoólica em adolescentes e jovens escolares. Einstein (São Paulo), 14, 143-151. https://doi.org/10.1590/S1679-45082016AO3677

Moyo, S., \& Rusinga, O. (2017). Contraceptives: adolescents' knowledge, attitudes and practices. A case studyof rural Mhondoro-Ngezidistrict, Zimbabwe. African jornal of reproductive health, 21(1), 49-63. https://doi.org/10.29063/ajrh2017/v21i1.4

Nunes, B. K. G., Guerra, A. D. L., Silva, S. M., Guimarães, R. A., Souza, M. M. D., Teles, S. A., \& Matos, M. A. D. (2017). O uso de preservativos: a realidade de adolescentes e adultos jovens de um assentamento urbano. https://doi.org/10.5216/ree.v19.39041

Oliveira, L. M. F. T. D., Santos, A. R. M. D., Farah, B. Q., Ritti-Dias, R. M., Freitas, C. M. S. M. D., \& Diniz, P. R. B. (2019). Influência do tabagismo parental no consumo de álcool e drogas ilícitas entre adolescentes. Einstein (São Paulo), 17. https://doi.org/10.31744/einstein_journal/2019AO4377

Oliveira, P. C., Pires, L. M., Junqueira, A. L. N., da Silva Vieira, M. A., Matos, M. A., Caetano, K. A. A. \& de Souza, M. M. (2017). Conhecimento em saúde sexual e reprodutiva: estudo transversal com adolescentes. Revista Eletrônica de Enfermagem, 19. https://doi.org/10.5216/ree.v19.39926 
Research, Society and Development, v. 10, n. 16, e419101623779, 2021

(CC BY 4.0) | ISSN 2525-3409 | DOI: http://dx.doi.org/10.33448/rsd-v10i16.23779

Pereira A. S., Shitsuka, D. M., Parreira, F. J., Shitsuka, R. (2018). Metodologia da pesquisa científica. [free e-book]. Santa Maria/RS. Ed. UAB/NTE/UFSM.

Rodrigues, K. A., Souza, M. F. N. S., Vieira, M. L., Benício, M. M. S., \& Freitas, D. A. (2018). Gravidez e doenças sexualmente transmissíveis na adolescência. Arquivos Catarinenses de Medicina, 47(2), 212-225. http://www.acm.org.br/acm/seer/index.php/arquivos/article/view/337

Silva, A. F., \&Lopes, M. H. B. M. (2018). Uso de métodos anticoncepcionais entre adolescentes de ensino médio. Adolescencia e Saude, 15(2), 102-112. http://www.adolescenciaesaude.com/detalhe_artigo.asp?id=725\&idioma=Espanhol

Silva, A. D. S. N., Silva, B. L. C. N., Silva Júnior, A. F., da Silva, M. C. F., Guerreiro, J. F., \& de Araújo, A. D. S. C. (2015). Início da vida sexual em adolescentes escolares: um estudo transversal sobre comportamento sexual de risco em Abaetetuba, Estado do Pará, Brasil. Revista Pan-Amazônica de Saúde, 6(3), 8-8. https://doi.org/10.5123/S2176-62232015000300004

Sousa, C. S. P. D., Castro, R. C. M. B., Pinheiro, A. K. B., Moura, E. R. F., Almeida, P. C., \& Aquino, P. D. S. (2018). Adaptación transcultural y validación de la Condom Self-Efficacy Scale: aplicaciónen adolescentes y adultos jóvenes brasileños 1. Revista Latino-Americana de Enfermagem, 25. https://doi.org/10.1590/1518-8345.1062.2991

SRE. Superintendência Regional de Ensino de Uberaba (2019). Lista de Escolas da SRE Uberaba. sre.uberaba.sedine@educacao.mg.gov.br

Tavares, C. M., Schor, N., França Júnior, I., Diniz, S. G. (2009). Fatores associados à iniciação sexual e uso de preservativo entre os adolescentes da Ilha de Santiago, Cabo Verde, África Ocidental. Cad Saúde Pública, 25(9). https://doi.org/10.1590/S0102-311X2009000 900011

Valim, E. M. A., Dias, F. A., Simon, C. P., Almeida, D. V. D., \& Rodrigues, M. L. P. (2015). Utilização de preservativo masculino entre adolescentes de escolas públicas na cidade de Uberaba (MG), Brasil: conhecimentos e atitudes. Cadernos Saúde Coletiva, 23, 44-49. https://doi.org/10.1590/1414$462 \times 295432170648$

Xu, Y., Chen, X., Yu, B., Joseph, V., \& Stanton, B. (2017). The effectsof self-efficacy in bifurcating there lation ship of perceived benefit and cost with condom use among adolescents: a cusp catástrofe modeling analysis. Journal of adolescence, 61, 31-39. https://doi.org/10.1016/j.adolescence.2017.09.004

Young, H., Burke, L., \& Gabhainn, S. N. (2018). Sexual intercourse, age of initiation and contraception among adolescents in Ireland: findings fromthe Health Behaviour in School-aged Children (HBSC) Ireland study. BMC Public Health, 18(1), 1-17. https://doi.org/10.1186/s12889-018-5217-z

Zangirolami-Raimundo, J., Echeimberg, J. D. O., \& Leone, C. (2018). Tópicos de metodologia de pesquisa: Estudos de corte transversal. J Hum Growth Dev, 28(3), 356-60. https://doi.org/ 10.7322/jhgd.152198 